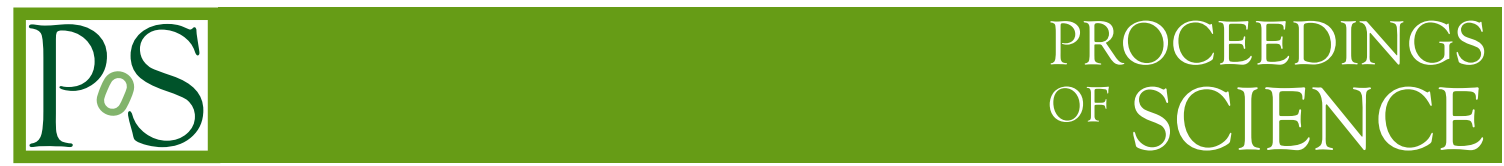

\title{
Search for compositeness and contact interactions in CMS
}

Kerstin Hoepfner* On behalf of the CMS Collaboration RWTH Aachen, III. Phys. Inst. A

E-mail: hoepfner@physik.rwth-aachen.de

A class of models assumes that fundamental particles have a substructure thus explaining existing observations as well as predicting new phenomena. The CMS experiment performed several searches for new physics resulting from such compositeness using data from pp collisions at $\sqrt{s}=7 \mathrm{TeV}$ (in 2011) and $\sqrt{s}=8 \mathrm{TeV}$ (in 2012).

36th International Conference on High Energy Physics,

July 4-11, 2012

Melbourne, Australia

${ }^{*}$ Speaker. 


\section{Search modes for compositeness with CMS}

The standard model (SM) of particle physics, albeit very successful, provides no explaination for the three generation structure of the fermion families. Attempts to explain the observed pattern have lead to a family of models postulating that quarks and leptons might be composite objects of fundamental constituents which are bound by an asymptotically free gauge interaction which becomes strong at a characteristic scale $\Lambda[1,2,3]$. Compositeness models predict the existence of excited quarks $\left(\mathrm{q}^{*}\right)$ and leptons $\left(\ell^{*}\right.$ such as $\mathrm{e}^{*}$ and $\left.\mu^{*}\right)$. Since they couple to ordinary SM fermions, they can be produced via contact interaction (CI) in collider experiments and subsequently decay radiatively to ordinary fermions through the emission of a W/Z boson or a photon.

The CMS [4] experiment has performed several searches for compositess using pp collisions at $\sqrt{s}=7 \mathrm{TeV}$ and $8 \mathrm{TeV}$ [5]. Rather generic searches look for deviations from the SM expectation in dijet, dilepton and lepton+MET distributions, complemented by searches for specific signatures which would oocur as a consequence of excited fermions, such as $\ell \ell \gamma$ or boosted Z-boson events. Here, the following searches are described:

- EXO-12-016 [6] Search for q* by looking for a generic bump in the dijet mass spectrum based on 2012 data

- EXO-11-025 [7] Search for $\mathrm{q}^{*}$ in highly boosted $\mathrm{Z} \rightarrow \mu \mu$ events produced via gauge boson exchange

- EXO-11-034 [8] Search for excited electrons and muons in processes like $\ell \ell \rightarrow \ell \ell \gamma$

- EXO-11-009 [9] Search for 4-fermion CI yielding an excess in the SM dimuon mass spectrum

- EXO-12-010 [10] Search for an excess in the $\mu \nu$-transverse mass spectrum

\section{Searches for excited quarks and leptons}

Several theories can be addressed with dijet analyses. Excited quarks $\left(\mathrm{q}^{*}\right)$ from $\mathrm{qg}$ fusion would appear as heavy resonances. A generic search is looking for new particles decaying to jet pairs using the dijet mass spectrum as measured by CMS with $4.0 \mathrm{fb}^{-1}$ of pp collision data at $\sqrt{s}=8 \mathrm{TeV}$ [6]. The dijet mass distribution of the two leading jets is measured after requiring the pseudorapidity separation of the two jets to satisfy $|\Delta \eta|<1.3$ with each jet inside the region $|\eta|<2.5$. The measured dijet mass spectrum is fitted with a smooth, empirical parameterization which well describes the prediction from simulated QCD dijet events. Narrow resonances with a width less than the dijet mass resolution would be found by this generic search, independent on particular model assumptions. The observed dijet mass spectrum is well described by the SM and excited quarks, which decay to qg, are excluded with masses $1 \mathrm{TeV}<\mathrm{m}\left(\mathrm{q}^{*}\right)<3.19 \mathrm{TeV}$. The compositeness scale is set to be equal to the mass of the excited quark.

Another search concerns the channel $\mathrm{q}^{*} \rightarrow \mathrm{qZ} \rightarrow q \mu^{+} \mu^{-}$[7], where an excited quark is produced irradiating a Z-boson to go into its ground state which corresponds to a SM quark. The excited quark can be produced via 4-fermion CI or via gauge boson exchange. Considering the 
Z-boson decaying into a pair of oppositely charged muons, the signal is constituted by two boosted (nearby) muons opposite a jet. The search is performed in $1 / \mathrm{p}_{\mathrm{T}}(\mu \mu)$ where the SM background is steeply falling to nearly zero at $1 / \mathrm{p}_{T}$-values around $0.001-0.002$ while a potential signal would yield clear peaks in that region (see Fig. 1). To determine the background, a template is fit to the high statistics and signal-free region corresponding to $\mathrm{p}_{T}=125-360 \mathrm{GeV}$ and extrapolating to high $\mathrm{p}_{T}$. The signal efficiency including acceptance is $50-70 \%$, increasing with $\mathrm{q}^{*}$ mass. An unbinned maximum likelihood fit for signal $\mathrm{L}_{\mathrm{s}-\mathrm{b}}$ and background $\mathrm{L}_{\mathrm{b}}$ is performed and no excess is found. 95\% confidence limit (CL) upper limits on the cross section of $\mathrm{q}^{*} \rightarrow \mathrm{qZ}$ for the benchmark models derived with the CLs limit setting criterion for the contact-interaction production. The results shown are obtained assuming all new couplings equal to the standard model couplings between ordinary fermions $\left(f=f \prime=f_{s}=1\right)$ and $\Lambda=M(q *)$. Exclusion limits are set for $\mathrm{q}^{*}$ production via 4-fermion interaction as $\mathrm{M}\left(\mathrm{q}^{*}\right)<2.14 \mathrm{TeV}$ (see Fig. 1-right) and via gauge boson exchange as $\mathrm{M}\left(\mathrm{q}^{*}\right)<1.94 \mathrm{TeV}$.
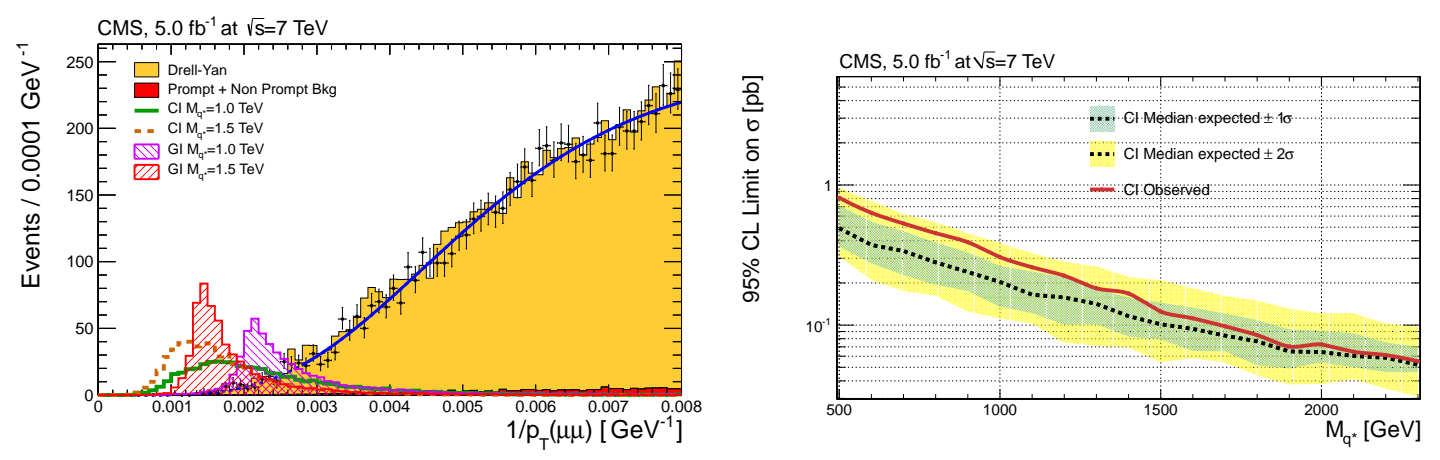

Figure 1: Search for excited quarks. Left: Distribution of $1 / \mathrm{p}_{T}$ for data (points with error bars), simulated SM backgrounds (stacked histograms), and simulated signal models (overlaid histograms). The signal models use either gauge interactions (GI) or contact interactions (CI). The blue solid line corresponds to the analytical template fit in the region defined as $0.0028 \mathrm{GeV}^{-1}<1 / \mathrm{p}_{T}<0.008 \mathrm{GeV}^{-1}$. Right: $95 \% \mathrm{CL}$ upper limits on the cross section of $\mathrm{q}^{*} \rightarrow \mathrm{qZ}$ via contact interaction derived assuming all new couplings equal to the SM couplings between ordinary fermions.

If excited states of electrons and muons exist at the new binding scale $\Lambda$, they can be produced via $\mathrm{CI}$ in association with an oppositely charged lepton. The excited lepton deay $\ell^{\star} \rightarrow \ell \gamma$ results in the final state $\ell^{+} \ell^{-} \gamma$, a search which was performed by CMS with $5 \mathrm{fb}^{-1}$ of data at $\sqrt{s}=7 \mathrm{TeV}$ [8]. After applying a veto on the Z-peak $(91 \pm 25 \mathrm{GeV})$, the two highest $\mathrm{p}_{T}$ leptons are chosen. Pairing each of them with the photon, a minimum $\left(M_{\ell \gamma}^{\min }\right.$, see example in Fig. 2-left) and a maximum invariant mass $\left(M_{\ell \gamma}^{\max }\right)$ are reconstructed. Both of them form a two-dimensional plane where the $\ell^{*}$ signal is represented in a L-shape region as seen in Fig. 2-right, with high invariant masses depending in detail on the $\ell^{*}$ mass. The dominant, irreducible, SM background in this search is the Drell-Yan production $\ell^{+} \ell^{-} \gamma$ where the final state photon is either due to initial or final state radiation. The second most important background is due to $\mathrm{Z}+\mathrm{jets}$ events where the jet is misidentified as a photon (or as an electron). To suppress the background, which clusters are lower invariant masses as also seen in Fig. 2-right, the signal search region is selected by two sets of upper and lower cuts on each of the invariant masses. After all selection steps the expected 
background is always below 2.5 events and decreases with $\ell^{*}$ mass while no indication for a signal is observed. Production cross sections higher than $1.60-1.35 \mathrm{fb}(1.30-1.14 \mathrm{fb})$ are excluded at the $95 \% \mathrm{Cl}$ for $\mathrm{e}^{*}\left(\mu^{*}\right)$ masses ranging from 0.6 to $2 \mathrm{TeV}$ correspondly excluding masses of excited electrons(muons) below 1.9(1.9) $\mathrm{TeV}$ for the scale of CI $\Lambda=M_{\ell^{*}}$.
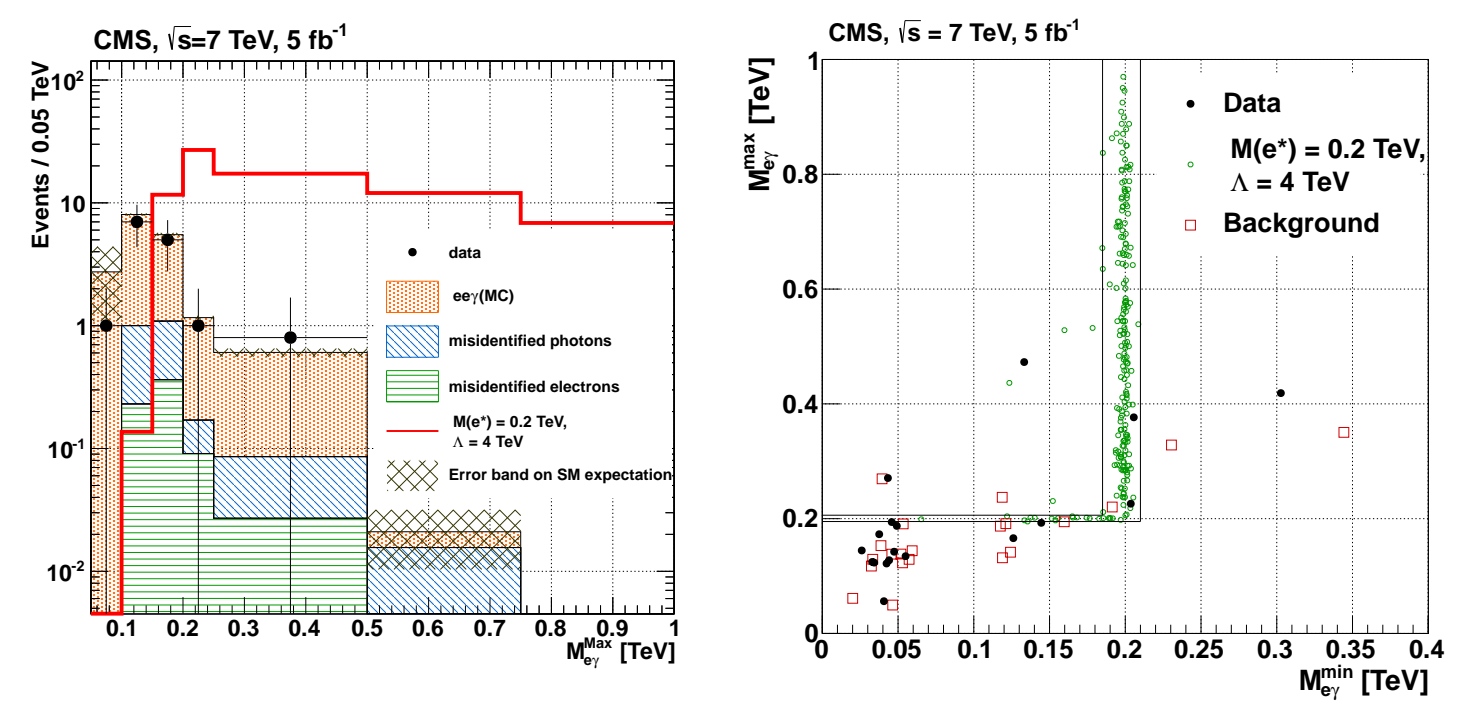

Figure 2: Search for excited leptons. Left: maximum invariant mass $M_{\ell \gamma}^{\max }$, here for the example of the electron channel. In addition to real ee $\gamma \mathrm{SM}$ events, misidentified photons and electrons contribute to the background. An example signal is shown for excited electrons of $200 \mathrm{GeV}$ mass and at $\Lambda=4 \mathrm{TeV}$. Right: two-dimensional plane formed by the minimum $\left(M_{\ell \gamma}^{\min }\right)$ and maximum $\left(M_{\ell \gamma}^{\max }\right)$ invariant mass showing the signal for a $200 \mathrm{GeV} \mathrm{e}$, SM background and the data.

\section{Four-fermion contact interactions}

A four-fermion interaction could lead to dimuon pairs which would contribute additional events to the high mass tail of the SM distribution. The SM processes being mainly DY/Z $\rightarrow \mu \mu$ with additional contributions from ttbar and diboson processes. The signal would manifest itself as an unstructured excess in the high mass tail of the dimuon spectrum, with its cross section depending on the new interaction scale $\Lambda$, as shown for two examples in Fig. 3-left. Since both processes, CI and SM SY, have the same final state, the scattering amplitudes add up and interfere either constructively or destructively. A search has been performed based on $5.3 \mathrm{fb}^{-1}$ of pp collision data at $\sqrt{s}=7 \mathrm{TeV}$ [9]. The data are interpreted in the context of a quark and muon compositeness model with a left-left isoscalar contact interaction described by an energy scale parameter $\Lambda$. No significant excess was found and 95\% CL exclusion limits are set of $\Lambda>9.5(13) \mathrm{TeV}$ for four-fermion CI with constructive (destructive) interference [9]. The limit with the one and two sigma uncertainty band as a function of the invariant mass is shown in Fig. 3-right.

In the helicity non-conserving (HNC) CI model [11], the final state consists of a charged lepton and a neutrino. This search has been performed in the $\mu \nu$ channel [10], where no experimental limit for this particular model exists yet, using $3.8 \mathrm{fb}^{-1}$ of pp data at $\sqrt{s}=8 \mathrm{TeV}$. The transverse 
mass $\left(\mathrm{M}_{\mathrm{T}}\right)$ is reconstructed from the transverse momentum of the muon and the missing transverse energy and the signal would yield an excess as seen for three values of Lambda in Fig. 4-left. The signal efficiency including acceptance is about $78 \%$ and nearly independent on Lambda. The spectrum is analysed by integrating all events (signal as well as background) above a lower $\mathrm{M}_{\mathrm{T}}$ threshold (around $1 \mathrm{TeV}$ ) which was optimized for the best expected limit. The data and the total expected SM background are shown in Fig. 4-right. No significant excess is observed and a 95\% CL exclusion limit of $8.7 \mathrm{TeV}$ is being set based on $3.7 \mathrm{fb}^{-1}$ of pp collision data at $\sqrt{s}=8 \mathrm{TeV}$ [10].
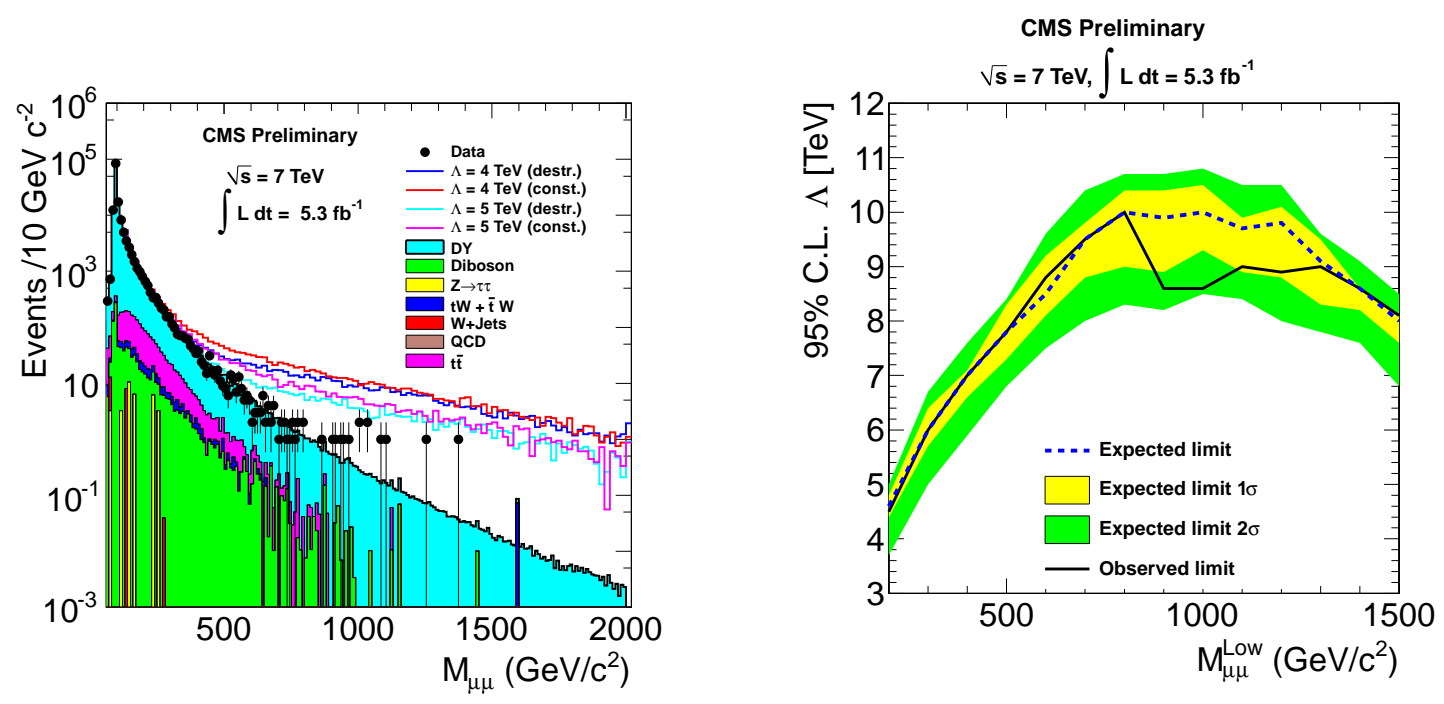

Figure 3: Search for 4-fermion contact interaction. Left: Dimuon invariant mass spectrum showing the expected SM background, two example signals for $\lambda=4 \mathrm{TeV}$ and $5 \mathrm{TeV}$ (constructive and destructive interference for each case) along with the data. Right: 95\% CL exclusion limit of $\Lambda<13 \mathrm{TeV}$ for the case of constructive interference. For destructive interference values of the compositeness scale $\Lambda<9.5 \mathrm{TeV}$ are excluded.
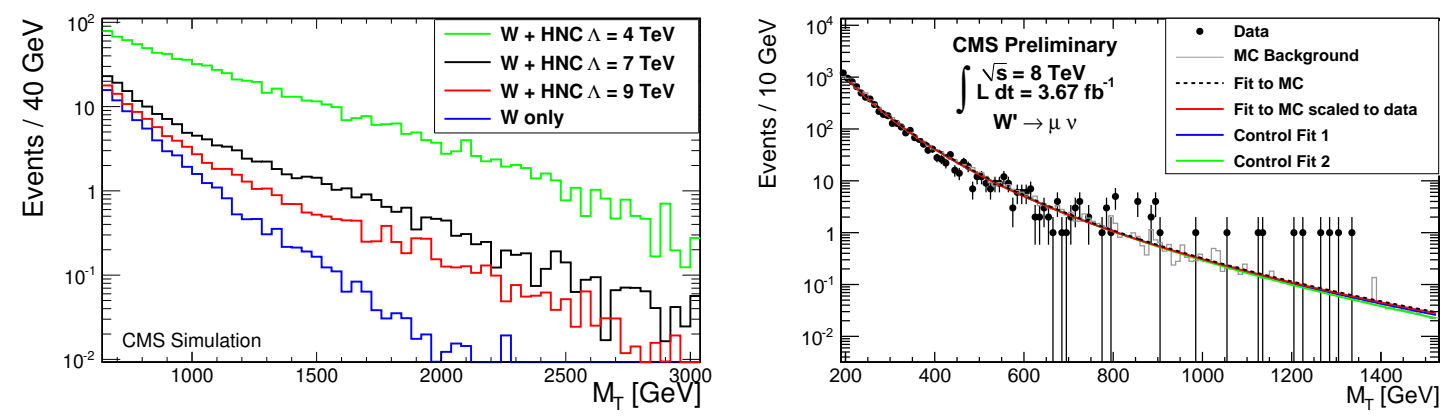

Figure 4: Search for CI in the HNC model. Left: Transverse mass spectrum $\left(\mathrm{M}_{T}\right)$ based on the $\mathrm{p}_{T}$ of muons and MET. Shown are a simulation of the main SM background which is the process $W \rightarrow \mu \nu$ along with three potential signals for the HNC model. Right: The expected SM background in the transverse mass distribution is determined from simulation of all relevant SM processes and a fit to this distribution. Also shown are the data points which are well described by the SM expectation. 


\section{Summary}

Several searches for compositeness and CI are ongoing in CMS [5]. First results are already based on the 2012 data at $\sqrt{s}=8 \mathrm{TeV}$. No indications for compositeness are yet seen and limits on excited fermions are set up to masses to $3 \mathrm{TeV}$. Limits on CI reach up to $13 \mathrm{TeV}$, depending on the model and the mode.

\section{References}

[1] H. Terazawa, M. Yasue, K. Akama and M. Hayashi: Observable Effects of the Possible Substructure of Leptons and Quarks, Phys. Lett. B112 (1982), p.387, arXiv hep-ph/1110.0713v1

[2] U. Baur, I. Hinchliffe, and D. Zeppenfeld: Excited Quark Production at Hadron Colliders, Int. J. Mod. Phys. A 2 (1987) 1285, doi:10.1142/S0217751X87000661.

[3] U. Baur, M. Spira, and P. M. Zerwas: Excited Quark and Lepton Production at Hadron Colliders, Phys. Rev. D 42 (1990) 815, doi:10.1103/PhysRevD.42.815.

[4] CMS Coll.: The CMS experiment at the CERN LHC, JINST 2008, volume3, S08004, doi "10.1088/1748-0221/3/08/S08004"

[5] https : //twiki.cern.ch/twiki/bin/view/CMSPublic/PhysicsResultsEXO

[6] CMS coll.: Search for narrow resonances using the dijet mass spectrum in pp collisions at $\operatorname{sqrt}(\mathrm{s})=8 \mathrm{TeV}$, CMS-PAS-EXO-12-016

[7] CMS Coll.: Search for anomalous production of highly boosted $\mathrm{Z}$ bosons decaying to dimuons in pp collisions at sqrt(s)=7TeV, CMS-PAS-EXO-11-025, CERN-PH-EP-2012-294, arXiv:1210.0867 (hep-ex)

[8] CMS coll.: Search for excited leptons in pp collisions at sqrt(s)=7TeV, CMS-PAS-EXO-11-034, CERN-PH-EP-2012-261, arXiv:1210.2411 (hep-ex)

[9] CMS Coll.: Search for evidence of contact interactions in dimuon mass spectrum, CMS-PAS-EXO-11-009

[10] CMS Coll.: Search for leptonic decays of $\mathrm{W}^{\prime}$ bosons in pp collisions at sqrt(s) $=8 \mathrm{TeV}$, CMS-PAS-EXO-12-010

[11] K.D. Lane, F.E. Paige, T. Skwarnicki and W.J. Womersley: Simulations of supercollider physics, Phys.Rept., volume 278 (1997), p.291, arXiv hep-ph/9412280, BNL-61138, BUHEP-94-31, FERMILAB-PUB-94-392-E 